\title{
Control of the receding meniscus in immersion lithography
}

\author{
H. Burnett, T. Shedd, ${ }^{\text {a) }}$ G. Nellis, M. El-Morsi, and R. Engelstad \\ Computational Mechanics Center, University of Wisconsin, 1513 University Avenue, Madison, Wisconsin \\ 53706
}

S. Garoff and K. Varanasi

Interfacial Physics Group, Carnegie Mellon University, Pittsburgh, Pennsylvania 15213

(Received 1 June 2005; accepted 29 August 2005; published 1 December 2005)

\begin{abstract}
The implementation of immersion lithography requires a sophisticated fluid management system. The design of the fluid management system must simultaneously consider liquid heating, viscous shear, normal forces, air entrainment, and the control of the liquid/air interfaces. In particular, it is becoming clear that the behavior of the receding air/water interface is critical and must be carefully controlled in order to prevent the deposition of residual liquid due to film pulling or the interface instability referred to as meniscus overflow. This paper discusses these failure mechanisms that are associated with the receding meniscus and that have been experimentally observed. In addition, a simple yet physics-based engineering model of the receding meniscus failure is presented and the results of the model are compared to experimental data. (C) 2005 American Vacuum Society.
\end{abstract}

[DOI: $10.1116 / 1.2090968]$

\section{INTRODUCTION}

The implementation of immersion lithography relies on successful management of the immersion fluid. The immersion fluid must be confined to a region very near the lens and must also allow the wafer to scan under the lens during exposure. These requirements imply the existence of water/air interfaces that are bounded at the end points by advancing and receding contact lines, as shown in Fig. 1. Control of these interfaces is difficult due to the rapid movements and accelerations of the substrate. This paper investigates the failure of the receding meniscus, an event that will result in residual liquid left on the wafer in the form of a thin film or droplets. Liquid droplets on the wafer may impact the advancing meniscus during subsequent scans and thereby generate air bubbles in the under lens region. The evaporation of a residual liquid film will result in the deposition of any contaminants contained in the liquid. Either of these processes may result in imaging defects during subsequent scans.

The receding meniscus may fail either due to the onset of film pulling or the less well-known phenomenon that is referred to as meniscus overflow. Film pulling causes a gradual failure of the receding meniscus and results in relatively small volumes of residual liquid deposited on the wafer. The contact angle that characterizes the intersection of the receding meniscus with the wafer is reduced as the wafer velocity increases. When the contact angle approaches zero, a very thin film of liquid will be "pulled" from the receding meniscus. This has been experimentally observed, as shown in Fig. 2(a), and is well documented in the literature ${ }^{1-3}$ film pulling is the mechanism exploited by many coating technologies to apply a thin, uniform film of liquid to a surface.

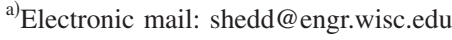

A catastrophic failure of the receding meniscus that results in the deposition of large amounts of immersion fluid on the wafer has been identified and is referred to as meniscus overflow. Meniscus overflow occurs when the dynamic pressure rise that is induced through the lens-wafer gap due to the wafer motion cannot be resisted by the surface tensioninduced pressure jump associated with the advancing and receding interfaces. The shape of these interfaces will adjust under the influence of the applied velocity-driven pressure force; the meniscus shape is altered as it assumes an increasingly small radius of curvature in order to produce a larger pressure jump. Eventually, the radius of curvature reaches the minimum possible value given the geometry of the system and the contact angle of the surfaces at which point any further increase in velocity will result in a failure of the receding interface. When meniscus overflow occurs, the liquid is carried out of the gap by the wafer rather than adhering to the lens, as shown in Fig. 2(b). Meniscus overflow results

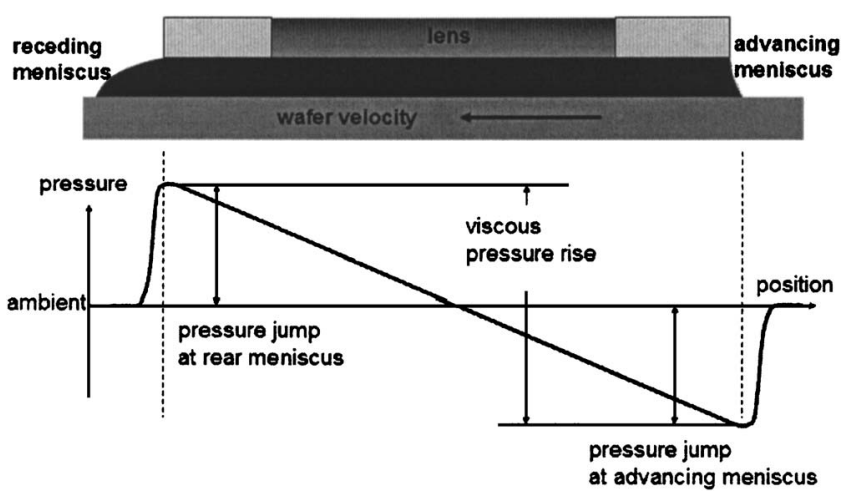

FIG. 1. Side-view schematic of a fluid management system and associated pressures. 


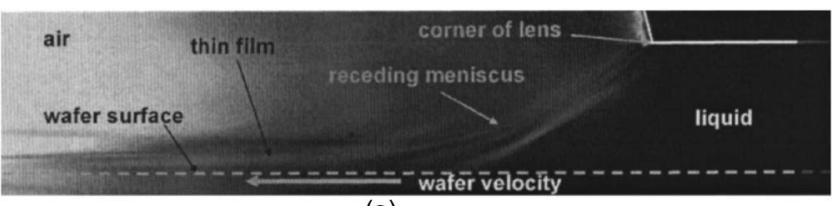

(a)

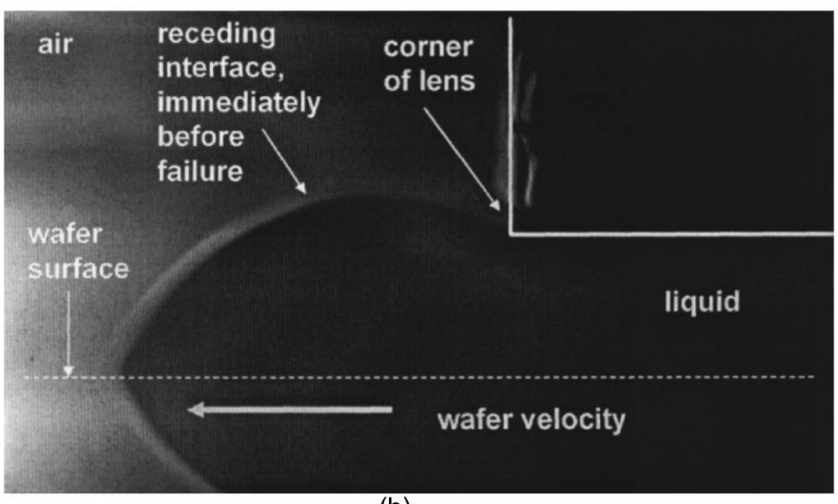

(b)

FIG. 2. Images of (a) film pulling and (b) meniscus overflow failures.

in bulk fluid loss from the lens-to-wafer gap and is aggravated by large wafer acceleration and deceleration and high wafer velocity.

In order to develop a better understanding of these receding meniscus failure mechanisms, an experimental apparatus has been fabricated in which a circular lens is placed in close proximity to a moving stage, and the critical velocity associated with receding meniscus failure was measured as a function of gap height. In addition, an engineering model of the meniscus instability will be presented that has been developed by combining computational fluid dynamics (CFD) and finite difference models of the flow in the gap (steadystate and transient) with CFD and geometric models of the shape of a pinned free surface.

\section{EXPERIMENTAL SETUP}

A system with a nominally static and stationary meniscus was constructed in order to study meniscus overflow and film pulling. The test facility is illustrated in Fig. 3 and consists of an adjustable gap between a test surface that is affixed to a motorized linear stage and a $5.1 \mathrm{~cm}$, circular quartz window that is mounted to a vertical stage positioned with a digital micrometer. For ease of fabrication, the facility was constructed such that the test surface is above the window, that is, "upside-down" with respect to the previous illustrations. At the gap heights and velocities investigated, changes in orientation with respect to gravity did not appear to modify the observed meniscus behavior, which is consistent with the very small Bond number $\left(\mathrm{Bo}=g p h^{2} / \gamma, \sim 0.1\right.$ for $h=1.0 \mathrm{~mm}$ ) characterizing the experiment.

Water was manually injected between the test surface and the quartz window prior to acquiring each data series using a syringe and injection needle. Meniscus behavior was observed for a range of gap heights and velocities by imaging the meniscus edge with a high-speed camera through a mi-

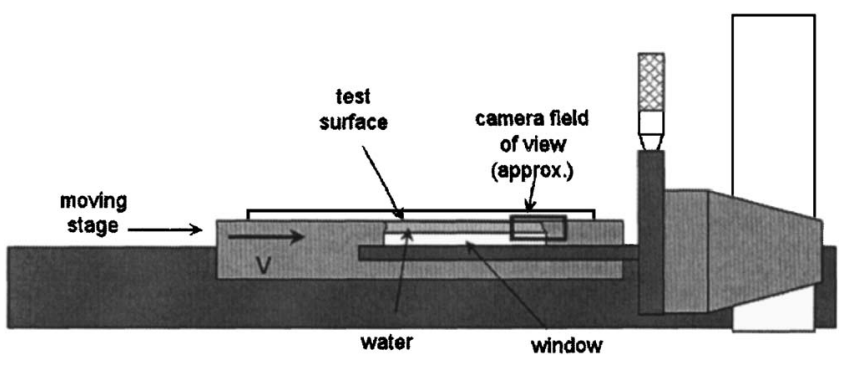

FIG. 3. A schematic of the test facility for study of meniscus behavior.

croscope objective as the substrate pulled out of the fluid. Steady-state and transient (accelerating and decelerating) behavior was recorded. The test surface and window were matched: when using a Rain-X®-treated test surface, the window was also treated with Rain- $X ®$; likewise, when using a quartz test surface, an untreated quartz window was used.

\section{EXPERIMENTAL RESULTS}

\section{A. Quartz substrate}

Images were acquired at various velocities and gap heights with a quartz substrate. This substrate was a $150 \mathrm{~mm} \times 150 \mathrm{~mm}$ square, $6 \mathrm{~mm}$ thick piece of clean quartz. In between each trial, the surface was cleaned with clean, dry air, then application of a commercial lens cleaning solution, and finally a methanol wipe performed with the "drop and drag" method. This resulted in static a consistent static contact angle of $41.5^{\circ} .{ }^{4}$ The substrate velocity was varied from 1 to $800 \mathrm{~mm} / \mathrm{s}$, while the gap height was varied from 0.25 to $2.0 \mathrm{~mm}$. Results depicting the observed qualitative behavior of the meniscus are summarized in Fig. 4 as a function of velocity and gap height. The transition to film pulling occurs abruptly at a velocity of $20-30 \mathrm{~mm} / \mathrm{s}$, and this transition appears to be less dependent on gap height than on velocity; this is consistent with the critical velocity for film pulling predicted by film-pulling models found in the literature [Eq. (9)]. In the region exhibiting no meniscus overflow, for a given velocity the meniscus radius of curvature increases and the overall meniscus slope decreases with increasing gap height. This agrees with theory, which predicts a decreasing radius of curvature in order to resist the increased viscous pressure that is generated by higher velocities. For a given gap height, the meniscus slope becomes more horizontal with increasing velocity. The increasing velocity "pulls" the fluid out further, flattening the meniscus slope. The meniscus tends to be concave in shape when it is contained between two hydrophilic surfaces. Only at high velocities and gap heights does the concavity reverse, at which point the bulging behavior related to the onset of meniscus overflow is observed.

\section{B. Rain-X-treated glass substrate}

Images were acquired at various velocities and gap heights with a Rain-X®-treated glass substrate. The 


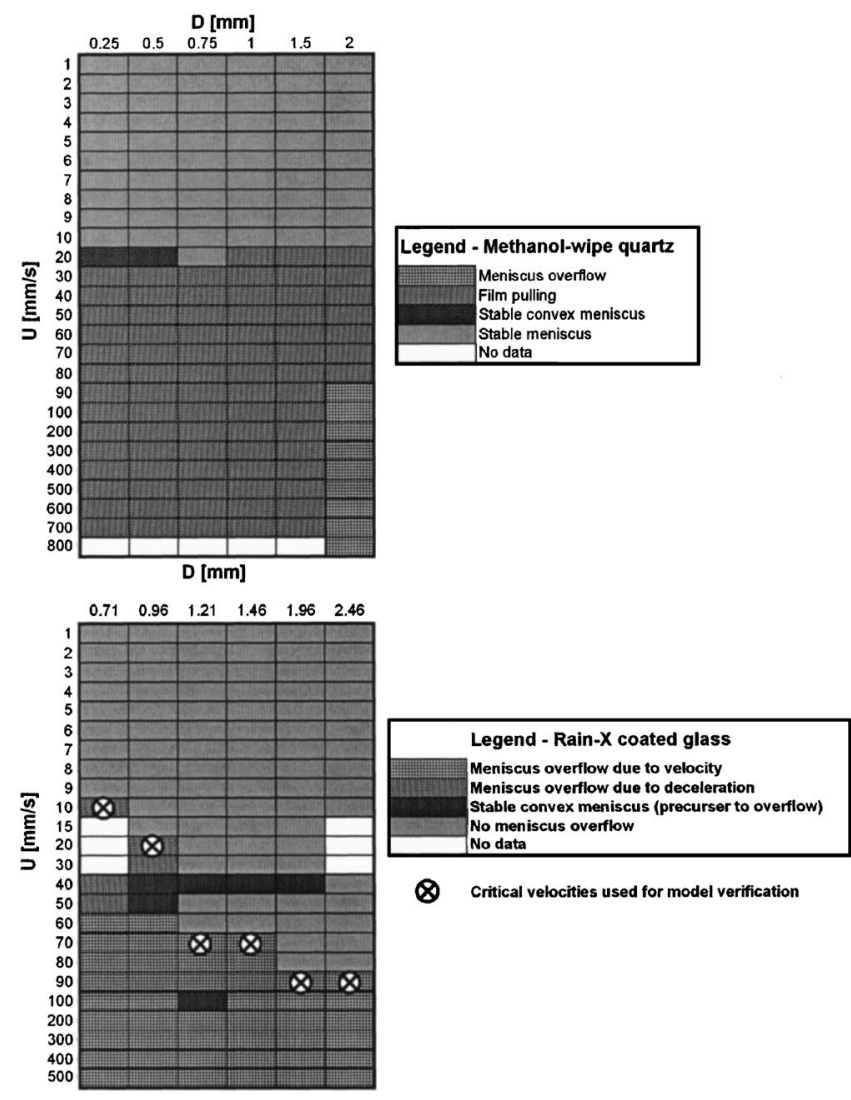

FIG. 4. Graphical illustration of experimentally determined critical velocities for flow over (top) a quartz substrate and (bottom) Rain-X®-treated glass.

Rain-X® was re-applied according to the manufacturer's recommendations between each set of trials at a given velocity and gap height. This provided a consistent static contact angle of $90.0^{\circ}{ }^{4}$ The substrate velocity was varied from 1 to $500 \mathrm{~mm} / \mathrm{s}$, while the gap height ranged from 0.71 to $2.46 \mathrm{~mm}$. A summary of the observed qualitative behavior of the receding meniscus can be seen in Fig. 4 as a function of the velocity and gap height. The meniscus behavior transitions from no meniscus overflow to meniscus overflow within the velocity range of 30 to $200 \mathrm{~mm} / \mathrm{s}$ for the gap heights investigated. There is a compromise between small gap height and high velocity, as stable flow can be sustained at higher velocities when the gap height is larger. This observation is consistent with the critical velocity that is predicted by the engineering model [Eq. (1)].

\section{ENGINEERING MODEL FOR MENISCUS OVERFLOW}

Meniscus overflow occurs when the liquid in the lenswafer gap does not remain adhered to the lens but rather tends to be pulled out of the gap by the wafer motion. The approximate pressure distribution within the meniscus is shown in Fig. 1. Note that the pressure tends to rise in the direction of the wafer velocity. This requires a subambient pressure at the right-hand side and a pressure above ambient at the left-hand side of the liquid volume; this difference in pressure between the fluid and ambient on either side must be sustained by the surface tension, which is reflected by changes in radius of curvature of the meniscus.

The shear-driven flow due to the wafer motion requires that there be a net fluid flow from right to left; therefore, a pressure gradient is generated that forces an equal flow from left to right in order to satisfy continuity. An interface instability that leads to meniscus overflow occurs when the front and receding interfaces cannot achieve a sufficiently small curvature in order to resist this viscous pressure rise. The critical velocity occurs when the largest possible pressure jumps at the rear and advancing menisci are exactly balanced by the viscous pressure rise. At steady state, this condition can be expressed as

$$
\frac{\gamma}{r_{r}}+\frac{\gamma}{r_{a}}=K_{v} \frac{\mu D V_{w}}{h^{2}},
$$

where $\gamma$ is the fluid surface tension, $r_{r}$ and $r_{a}$ are the radii of curvature of the receding and advancing interfaces, respectively, $h$ is the gap height, $\mu$ is the viscosity of the fluid, $D$ is the diameter of the lens, and $V_{w}$ is the wafer velocity. The parameter $K_{v}$, the viscous pressure drop factor, is a dimensionless constant that depends on the configuration of the flow passage. The value of $K_{v}$ was determined for the threedimensional, circular gap typical of an immersion system using computational fluid dynamics (CFD) simulations, as described in the subsequent section. Equation (1) is valid only for steady-state wafer motion and there may be a significant additional pressure rise that is related to inertial effects.

\section{A. Steady viscous pressure rise model}

Fully developed flow through an infinitely wide gap with length $L$ and height $h$ in which one surface is moving with velocity $V_{w}$ is characterized by

$$
\frac{Q}{w}=\frac{V_{w} h}{2}-\frac{\Delta P h^{3}}{12 \mu L},
$$

where $Q / w$ is the volumetric flow rate per unit width of gap and $\Delta P$ is the pressure drop across the gap. In the absence of any net flow through the gap, Eq. (2) simplifies to

$$
\Delta P=\frac{6 L \mu V_{w}}{h^{2}} .
$$

Equation (3) indicates that a pressure rise is induced across the length of the gap due to wafer motion. This pressure rise is proportional to the product of the gap length, plate velocity, and the fluid viscosity and inversely proportional to the gap height squared. In the simple case of an infinitely wide, two-dimensional gap, the constant of proportionality is exactly 6.0. However, for the more complex situation associated with flow driven laterally across a circular lens-wafer gap, the constant of proportionality must be determined through CFD simulations.

The viscous pressure drop factor $K_{v}$ is defined based on the scaling identified in Eq. (3): 


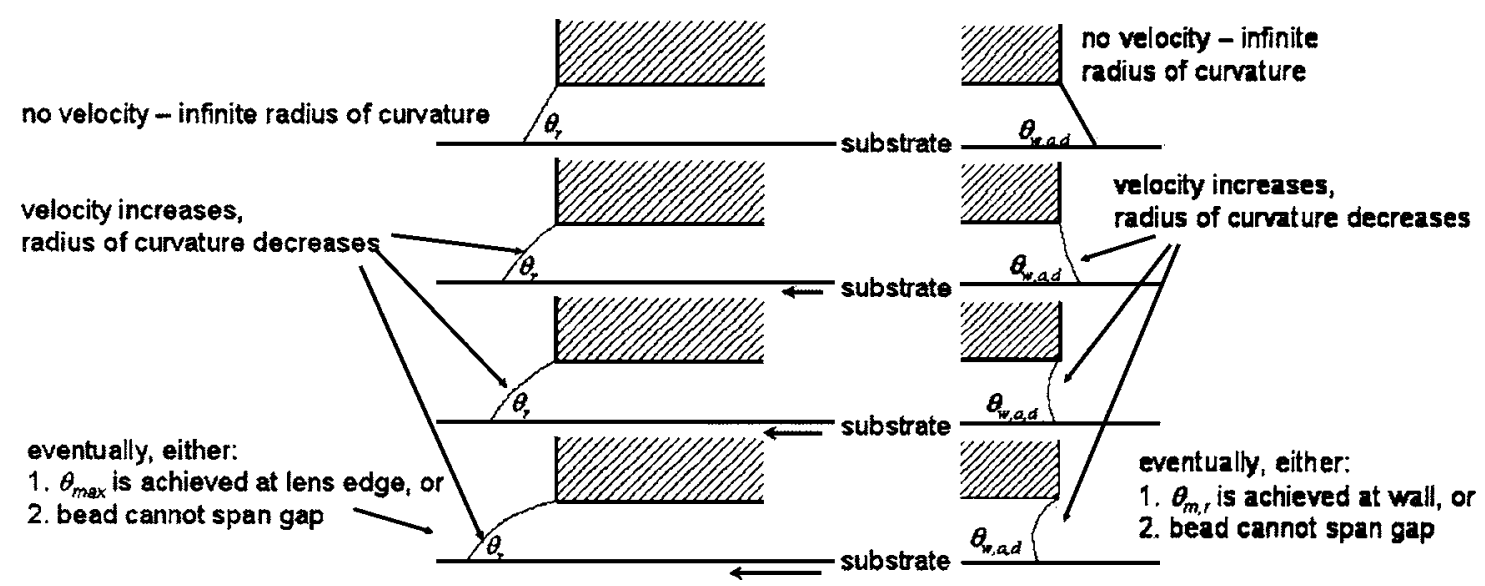

FIG. 5. Schematic of the behavior of (left) the receding interface adjusting to an increasing pressure within the fluid due to the wafer velocity and (right) the advancing meniscus shape as velocity increases.

$$
K_{v}=\frac{h^{2} \Delta P}{D \mu V_{w}} .
$$

The viscous pressure drop factor associated with the CFD simulation results was calculated at each condition and it was found that $K_{v}=4.75$ adequately represents the steady-state pressure rise for the conditions associated with immersion lithography.

\section{B. Meniscus shape model}

Meniscus overflow occurs when the fluid escapes from the fluid management system gap because the viscous pressure rise overwhelms the stabilizing surface tension force. This occurs when geometric limitations on the radius of curvature prevent any further reduction without reaching a condition where the meniscus is released from its pinned state. The critical velocity is the velocity at which this takes place.

Figure 5 illustrates the progression of the interface shape as the velocity approaches the critical velocity. Initially, at very low velocity, there is no pressure rise across the lensto-wafer gap and therefore no countering surface tension force is required. The meniscus radii of curvature on both the receding and advancing sides approach infinity and the interface becomes a straight line that stretches from the pinning point at the edge of the fluid management surface and the wafer, and satisfies the required receding contact angle at the wafer surface, $\theta_{r}$.

As the velocity increases, the meniscus on both sides is deformed so that there is a pressure jump across the meniscus, as shown in Fig. 1. The increased pressure jump results in a reduction in the radius of curvature such that the interface is convex as seen by the fluid. The result is a gradual bowing out of the meniscus, as shown in Fig. 5.

Eventually, the pinned contact line at the surface of the receding side of the fluid management system may achieve the static advancing contact angle with respect to the surface along the side of the fluid management system where the interface will be released from the pinning corner and climb up the side. This immediately results in an increase in the radius of curvature and therefore meniscus overflow. The maximum ratio of gap height to radius of curvature that can be achieved in this limit is

$$
\frac{h}{r_{r}}=\cos \left(\theta_{m, a}-\frac{\pi}{2}\right)-\sin \left(\frac{\pi}{2}-\theta_{w, r, d}\right)
$$

where $\theta_{m, a}$ is the static advancing contact angle associated with the fluid management system surface and $\theta_{w, r, d}$ is the dynamic receding contact angle associated with the wafer.

Alternatively, the fluid meniscus may be unable to both retain a circular shape and span the gap, in which case it becomes stretched; this also results in an increase in the radius of curvature and meniscus overflow. The maximum ratio of gap height to radius of curvature that can be achieved in this limit is

$$
\frac{h}{r_{r}}=1-\sin \left(\frac{\pi}{2}-\theta_{w, r, d}\right)
$$

Equations (5) and (6) describe the surface tension force that can be achieved at the receding meniscus. The advancing meniscus also plays a role in meniscus overflow, as indicated by Eq. (1). Figure 5 illustrates how the shape of the advancing meniscus is affected by increasing velocity.

As the velocity increases, the advancing meniscus changes shape from linear (i.e., having an infinite radius of curvature) to convex, as viewed from the liquid. Eventually, either the static receding contact angle is achieved at the lens surface or the interface cannot maintain a circular shape and span the gap. In the first limit, the ratio of the gap height to radius of curvature is given by

$$
\frac{h}{r_{a}}=\sin \left(\frac{\pi}{2}-\theta_{m, r}\right)+\sin \left(\frac{\pi}{2}-\theta_{w, a, d}\right),
$$

where $\theta_{m, r}$ is the static receding contact angle associated with the fluid management system surface and $\theta_{w, a, d}$ is the dynamic advancing contact angle associated with the wafer. In the second limit, the ratio of the gap height to radius of curvature is given by 


$$
\frac{h}{r_{a}}=1+\sin \left(\frac{\pi}{2}-\theta_{w, a, d}\right)
$$

Equations (5)-(8) were derived based on geometry and fail to account for the effect of fluid pressure or flow on the interface shape beyond their impact on the dynamic contact angles. To verify that these equations adequately capture the surface tension force, CFD models were generated. The CFD models considered only the local region very near the interface (analogous to Fig. 5) and considered the case where the gap is initially filled with liquid that is pinned at the corner of the fluid management system. The pressure just inside the gap was gradually increased or decreased relative to ambient in order to simulate the receding or advancing meniscus, respectively. The pressure elevation at which the meniscus becomes unstable and overflows is compared to the pressure jump predicted by the minimum of Eqs. (5) and (6). The pressure reduction at which the meniscus becomes unstable and is pulled into the gap is compared to the pressure jump predicted by the minimum of Eqs. (7) and (8). Reasonable agreement was observed between the model and the CFD results.

\section{Film-pulling model}

The dynamic contact angle that characterizes the intersection of the receding meniscus with the wafer is reduced as the wafer velocity increases. When the dynamic contact angle approaches zero, a very thin film of liquid will be "pulled" from the receding meniscus. This behavior is most easily observed on hydrophilic surfaces. This failure mechanism is not as extreme as meniscus overflow; however, undesirable droplets of liquid are deposited on the substrate surface.

Several researchers have presented correlations that describe the variation of the dynamic contact angle with velocity. Immersion lithography will be characterized by relatively large Reynolds numbers and large accelerations, and therefore most previous work in the area of film pulling for coating technologies is not directly applicable. However, correlations for the receding dynamic contact angle under idealized and inertia-free conditions have been shown to be nominally consistent with CFD models that predict film pulling under conditions expected for immersion lithography. Therefore, it is possible to approximately predict the velocity at the onset of film-pulling using Voinov's equation ${ }^{1}$ by setting the dynamic contact angle to zero:

$$
V_{w}=\frac{\gamma \theta_{s, r}^{3}}{9 \mu\left[\ln \frac{h}{L_{s}}+C\right]},
$$

where $\gamma$ is the surface tension, $\theta_{s, r}$ is the static receding contact angle, $\mu$ is the viscosity of the fluid, $h$ is the halfwidth of the gap (nominally $0.5 \mathrm{~mm}$ for immersion lithography), and $C$ is a solution constant that is equal to 1.5 for a slot. $L_{s}$ is the slip length, which is related to the inner-scale physics of the meniscus and is typically expressed as $\mu / \beta$, where the slip coefficient $\beta^{-1}$ has a value of approximately

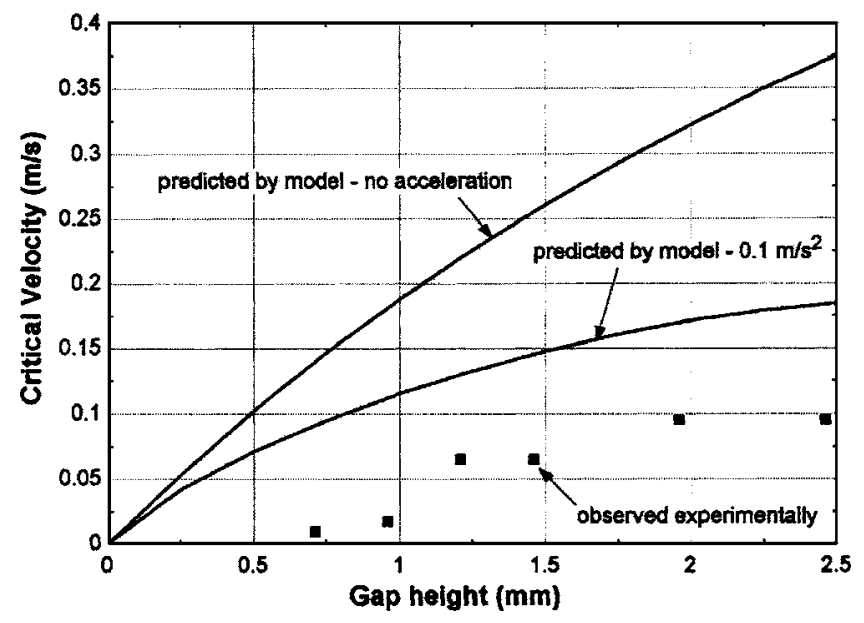

FIG. 6. Critical velocity for meniscus overflow as a function of gap height, measured and predicted.

$3 \times 10^{-4}$ to $1 \times 10^{-3} \mathrm{~cm}^{3} /$ dyn s. ${ }^{1}$ The exact value of the slip length has a very small effect on the dynamic contact angle. It is noted that Voinov's equation is strictly valid for vanishingly small Reynolds number flows, whereas a relatively large Reynolds number, on the order of 1000 , will characterize the flow conditions expected in immersion lithography. This may partially explain why Eq. (9) consistently overpredicts the velocity at the onset of film pulling when compared to experimental observations. However, the reason for this discrepancy is not well understood and is being investigated.

\section{COMPARISON BETWEEN EXPERIMENT AND ENGINEERING MODEL}

The critical velocity for meniscus overflow on Rain-Xtreated glass (the data points illustrated in Fig. 4) were plotted together with the engineering model predictions in Fig. 6. The engineering model was run with an advancing angle of $103.4^{\circ}$ and a receding angle of $92.2^{\circ}$ on both the substrate and window surfaces. The model predicts greater critical velocities than those observed experimentally; however, model predictions including the transient pressure gradient that is associated with $0.1 \mathrm{~m} / \mathrm{s}^{2}$ acceleration approach experimental results. This suggests that acceleration effects may have played a significant role in the experiment.

A high rate of acceleration or deceleration leads to an augmentation of the pressure gradient, which can lead to bulk fluid loss even when the steady-state behavior would correspond to film pulling or a stable convex meniscus. As the substrate decelerates, a bulb of fluid will accumulate at the contact line. In this work, deceleration-induced overflow was observed only on the quartz substrate. Acceleration can lead to a bulge that either stabilizes into a no overflow condition or leads to meniscus overflow. Acceleration-induced overflow was observed on both Rain-X-treated glass and quartz substrates.

Several uncontrolled experimental variables may have contributed to variability in the results. This experimental setup provides little control of the initial fill condition, and 
therefore the liquid volume and the precise location of the interfaces on the fluid management system may vary from one run to the next. Higher values of liquid volume trapped in the gap lead to meniscus overflow at lower velocities and gap heights. The initial shape of the meniscus when substrate motion commences is not controlled. That is, the substrate could have been moving in either direction just prior to a data run. The possible effect of this has not been quantified. Care was taken to ensure that the receding meniscus was pinned at the edge of the lens at the start of each duty cycle; however, the position of the interface after the start of the cycle was not controlled. Care was also taken to dry the lens edge after a meniscus overflow episode; any residual water on that surface would encourage bulk meniscus overflow on a subsequent run. The substrate cleanliness is also critical, as particles on the surface can lead to premature film pulling or fluid loss.

\section{CONCLUSIONS}

For the anticipated flow conditions and surface characteristics in immersion lithography, fluid deposition on the wafer surface is expected based on the experimental and modeling results described above. Meniscus overflow and thin-film pulling are the two principal mechanisms observed and both lead to fluid deposition on the surface. Thick-film pulling is an additional mechanism that has been observed but is not well understood. An engineering model has been developed and verified under a limited set of conditions; this tool can be applied to map critical velocity for bulk meniscus overflow and film pulling for various potential shower head designs. The engineering model can be used to by tool designers to ensure that fluid deposition on the wafer surface is avoided.

\section{ACKNOWLEDGMENTS}

The authors appreciate the financial, logistical and technical support provided by SEMATECH, Inc., and technical program, managers C. Van Peski and A. Grenville. Important insights into the instability problem were provided by Dr. S. Weinstein (Kodak Research Labs) and Dr. E. Rame (NASA National Center for Microgravity Research).

${ }^{1}$ J. G. Petrov, R. V. Sedev, and P. G. Petrov, Adv. Colloid Interface Sci. 38, 229 (1992).

${ }^{2}$ P. G. Petrov and J. G. Petrov, Langmuir 8, 1762 (1992).

${ }^{3}$ P.-G. de Gennes, F. Brochard-Wyard, and D. Quéré, Capillarity and Wetting Phenomena (Springer, New York, 2004).

${ }^{4}$ H. B. Burnett, Master's thesis, University of Wisconsin-Madison, 2005. 